\title{
Facilitating and Managing Transitions: An Imperative for Quality Care
}

Afaf Ibrabim Meleis

PhD, FAAN

Dean Emerita and Professor of Nursing and Sociology,

University of Pennsylvania.

Professor Emerita, University of California, San Francisco
Redalyc: http://www.redalyc.org/articulo.oa? id $=145257605001$

\section{Editorial}

Being admitted to a hospital, preparing for discharge, receiving a chronic illness diagnosis, transferring from intensive care to regular care, moving to a nursing home, receiving a grave diagnosis, losing a family member, facing an environmental disaster, graduating and starting a new professional position—all of these scenarios may appear different, but ask yourself, what might they all have in common? They are all changes that trigger a transition process. While change is an event or a situation that is well defined and static in time, transition is a process with a range of responses. During transitions, individuals, families and communities experience unfamiliar environments, sensations and emotions, and confront different levels of uncertainty about what may come next. They may have expectations, realistic or uninformed, and experience disruptions in their daily lives and routines, all of which are influenced by and affect their health and wellbeing.

How a person perceives the change, its meaning and the availability of support and resources profoundly influences experiences and responses. People are influenced by the preparation they receive for anticipated changes, as well as continuity of care throughout the process up until the results of the change are incorporated into their daily lives and identities (1).

These changes require a process of adjustment, recalibration and development of new skills and capacities to meet the new demands imposed by the changes. They also require mobilization of inner and outer resources and tangible and intangible support for their environment. Nurses are at the center of these changes and the process of transition, and have always supported individuals preparing for and facing changes. For example, when patients are admitted to hospitals, nurses assess their health and illness status and their knowledge of their illness, helping patients navigate the admission process, hospital routines, as well as clarifying the roles of the many team members who visit their beds. They find out what patients' expectations are, provide accurate information about what they might expect, teach them new skills to manage their new environments and illness demands and insure access to the needed resources. Nurses also provide similar information, skills and support during discharge, preparing individuals and families to manage their own care. In other words, they facilitate patients' transitions and empower them through clarity, information, skills and resources, continuing the healing and recovery processes and enhancing wellbeing and quality of life (2).

Families, significant others and caregiving institutions, where patients may be referred to upon discharge, require information about patients and about how to continue to provide needed care that leads to recovery or self-care management within the context of a patient's own experiences and responses. Facilitating transitions is a part and parcel of continuity of care. Providing continuity of care requires strategies, resources, skills and knowledge that is congruent with the different stages in recovery and healing, or in the process of acquiring 
new roles, such as becoming a new mother or father, taking on a new at-risk role, or coping with the loss of bodily functions, an organ or a family member (3).

Theories provide the framework for assessment, diagnosis and intervention. They also frame the goals and outcomes of care. Theories are the reservoir for new discoveries, new evidence and new knowledge. They provide the concepts and the language to describe the phenomenon that nurses encounter during caring processes. Theories help in ensuring the succinct communication of assessment, interventions or proposed policy changes that affect care (4). Providing care from a transitions perspective for patients, who are facing changes in their lives, whether because of natural growth and development, chronic illness or loss of bodily functions, limbs or organs, increases the probability of properly delivering and evaluating quality care.

Transitions Theory provides a map and a vehicle to understand, communicate and interpret what populations experience when faced with changes that affect wellbeing. It provides the framework to assess the nature of disconnections with valued relationships and changes in daily life routines. Examples include, what daily diabetes testing, weekly dialysis, a crying baby, monthly chemotherapy treatments or caring for a parent with Alzheimer's means to a person, what disruptions may these changes create in their life and what is the nature of the distress they experience? (5).

It also allows for assessment of deficits and opportunities in knowledge, skills, support and resources, as well as ways in which the change may have positively transformed a person's life. Transitions Theory prompts the assessment of critical points or turning points during the recovery and healing processes and deciphers which interventions are appropriate at each of those points. Critical points may be different for different populations and reflect the different nature and properties of the change. Critical points may occur when a patient receives a third dose of chemotherapy, takes a class on in home colostomy irrigation, requires a palliative care treatment or when a family member with a cognitive impairment wonders unattended outside of a home for the first time. Critical points may be different for the changes that different populations face. Transitions Theory prompts its users to assess the patient's level of awareness and understanding of the critical points, the effect of the change on daily life, the stages the patient may go through and the importance of becoming more attuned to interpreting responses at different stages and critical turning points in the recovery process and in new role acquisition.

The goals of the assessment are to provide the necessary care to enhance confidence and self-care, to ensure quality of life and to optimize access to and use of appropriate inner and outer resources that are congruent with the needs at critical points. Another goal is to plan interventions to help individuals successfully integrate the change in their self-identity. Viewing a change as a transition process values attention to the availability of family, community and society, as well as including them in intervention plans. For example, moving a child with special needs, such as chronic illness or autism, from pediatric care two adult care, requires a handoff that involves pediatricians, adult caregivers, other health care providers, family, schools and health care systems (6).

Using theory as a framework enhances the development of coherent and impactful programs of research. A program of research driven by Transitions Theory could be designed to uncover patients' experiences and responses to new diagnoses, levels of engagement and confidence in managing their care, as well as how these influence adherence and compliance with new treatment regimens and quality of life of individuals and their caregivers. Questions such as what are the determinants of a healthy transition process and outcomes, what are the most effective critical points for a particular nursing intervention and what environmental resources are most effective at different stages in the transition for effective chronic illness management, are all questions driven by a transitions framework, and the answers to which can refine and modify the theory, as well as the knowledge base for living with and managing transitions.

Facilitating transitions requires clarity in communication, continuity of care, management of patient environment and engagement, opportunities for debriefing and follow up and ensuring the involvement of a 
transition team. Facilitating transitions toward healthy outcomes assumes interdisciplinarity and functioning healthcare teams (7).

With the many changes populations face, such as increasing life expectancy, increasing non-communicable diseases, living longer with Parkinson's, Alzheimer's or cancer and being discharged earlier from hospitals, all of which require longer continuous care to be assumed by patients and families, there is an urgent need for nurses to provide the care processes that enhance healthy transitions and outcomes. Nurses are instrumental in facilitating transitions toward wellbeing and quality of life. Health care systems must include and implement policies that allow the appointment of transitions' nurses, or health care teams, to insure healthy processes and outcomes for the changes that patients and families experience.

\section{References}

1. Meleis AI. Role insufficiency and role supplementation: A conceptual framework. Nurs Res. 1975;24(4):264-71.

2. Meleis AI. Transitions theory. In: Smith M, Parker M. Nursing theories and nursing practice. 5 th ed. Philadelphia: FA Davis; Forthcoming.

3. Meleis AI, Trangenstein PA. Facilitating transitions: Redefinition of the nursing mission. Nurs Outlook. 1994;42(6):255-9.

4. Meleis AI. Theoretical nursing: Development and progress. 6th ed. Philadelphia: Walters Kluwer; 2018.

5. Meleis AI, Sawyer LM, Im EO, Messias DKH, Schumacher K. Experiencing transitions: An emerging middle-range theory. Adv Nurs Sci [internet]. 2000;23(1):12-28. Available at: https://www.insights.ovid.com/pubmed?pm id $=10970036$.

6. White PH, Cooley WC, American Academy of Pediatrics, American Academy of Family Physicians, American College of Physicians, \& Transitions Clinical Report Authoring Group. Supporting the health care transition from adolescence to adulthood in the medical home. Pediatrics. 2018;142(5). https://www.doi.org/10.1542/p eds.2018-2587.

7. Naylor M, Berlinger N. Transitional care: A priority for health care organizational ethics. Hastings Cent Rep. 2016;46:S39-42. https://www.doi.org/10.1002/hast.631.

Creative Commons CC BY 4.0 